\title{
Comparison of Endocardial Activation Times at Effective and Ineffective Ablation Sites Within the Pulmonary Veins
}

\author{
HUNG-FAT TSE, M.B.B.S., CHU-PAK LAU, M.D., WILLIAM KOU, M.D.,* \\ FRANK PELOSI, M.D.,* HAKAN ORAL, M.D.,* MICHAEL KIM, M.D.,* \\ GREGORY F. MICHAUD, M.D., * BRADLEY P. KNIGHT, M.D.,* \\ MAURO MOSCUCCI, M.D.,* S. ADAM STRICKBERGER, M.D.,* \\ and FRED MORADY, M.D.*
}

From the Division of Cardiology, Department of Medicine, University of Hong Kong, Queen Mary Hospital, Hong Kong; and the *Division of Cardiology, Department of Internal Medicine, University of Michigan Medical Center, Ann Arbor, Michigan

\begin{abstract}
Pulmonary Vein Arrhythmias. Introduction: Recent studies demonstrated that atrial arrhythmias may be generated within pulmonary veins. The purpose of this study was to compare the endocardial activation times at effective and ineffective ablation sites during radiofrequency catheter ablation of arrhythmias initiated or generated within pulmonary veins.

Methods and Results: Twenty-one of 28 patients without structural heart disease underwent successful ablation of $\mathbf{2 3}$ arrhythmogenic foci within a pulmonary vein. Electrograms were recorded at 75 pulmonary venous sites and categorized into three groups: 23 successful ablation sites; 28 unsuccessful target sites within an arrhythmogenic pulmonary vein; and 24 sites within nonarrhythmogenic pulmonary veins. The endocardial activation time of premature depolarizations arising at successful target sites was significantly earlier than at other sites. During premature depolarizations, an endocardial activation time of $\mathbf{- 7 5} \mathrm{msec}$ or earlier had a sensitivity of $\mathbf{8 3} \%$ and a specificity of $\mathbf{7 9 \%}$ for identification of a successful ablation site. Endocardial activation times earlier than -100 msec were recorded only at successful ablation sites, and endocardial activation times later than $\mathbf{- 3 0}$ msec were recorded only at sites within nonarrhythmogenic pulmonary veins. The presence of a split potential during sinus rhythm or premature depolarizations was not a specific indicator of a successful ablation site.

Conclusion: The endocardial activation times of premature depolarizations that arise within pulmonary veins and initiate atrial tachycardia/fibrillation are useful in identifying successful ablation sites. (J Cardiovasc Electrophysiol, Vol. 11, pp. 155-159, February 2000)
\end{abstract}

pulmonary vein, atrial fibrillation, radiofrequency ablation, mapping

Introduction

Recent studies showed that the pulmonary veins may be a source of arrhythmogenic foci that trigger or generate atrial tachycardia, atrial fibrillation, or atrial flutter. $^{1-4}$ The endocardial activation time of the depolarizations generated within the pulmonary veins may be helpful in guiding radiofrequency catheter ablation. However, no prior studies have quantitatively analyzed the endocardial activation times at pulmonary venous ablation sites. The purpose of this study was to compare

Address for correspondence: Fred Morady, M.D., Division of Cardiology, B1F245, University of Michigan Medical Center, 1500 East Medical Center Drive, Ann Arbor, MI 48109-0022. Fax: 734-9367026.

Manuscript received 8 August 1999; Accepted for publication 28 October 1999. the endocardial activation times at effective and ineffective target sites for ablation of arrhythmias arising in the pulmonary veins.

\section{Methods}

\section{Patient Population}

The study population consisted of 21 consecutive patients who underwent successful radiofrequency catheter ablation of 23 pulmonary venous arrhythmogenic foci. In the electrophysiology laboratory, the procedure was considered successful if the spontaneous premature depolarizations or tachycardias that were arising in a pulmonary vein were no longer present with or without infusion of isoproterenol at a rate of $2 \mu \mathrm{g} / \mathrm{min}$. An additional criterion for successful ablation was either the lack of symptomatic arrhythmias in the absence of antiarrhythmic drug therapy after a mean follow-up period of 
$10 \pm 3$ months (range 6 to 18 ) in 15 patients, or a recurrence of atrial tachycardia/fibrillation that was found during repeat electrophysiologic testing to be arising from a pulmonary vein other than the one in which the original target site was located in six patients. During the period of time that the 21 patients in this study underwent the catheter ablation procedures, catheter ablation of an arrhythmia thought to be arising in a pulmonary vein was attempted without success in another seven patients. The reasons for unsuccessful ablation in these patients included the inability to localize an arrhythmogenic focus, the presence of multiple foci, or a lack of arrhythmias during the ablation procedure.

There were 16 men and 5 women (mean age $43 \pm 13$ years). None of the patients had structural heart disease. Mean left ventricular ejection fraction was $0.63 \pm 0.06$, and mean left atrial diameter by echocardiography was $3.8 \pm 0.4 \mathrm{~cm}$. The clinically documented arrhythmia was atrial tachycardia in 2 patients, paroxysmal atrial fibrillation in 14 patients, and persistent atrial fibrillation that had been present for a mean of $19 \pm 24$ months in 4 patients. Except in the patients who had persistent atrial fibrillation, 24-hour ambulatory ECG recordings demonstrated frequent atrial premature depolarizations ( 1,000 per 24 hours) and runs of nonsustained atrial tachycardia or atrial fibrillation. The patients had been symptomatic for a mean of $52 \pm 57$ months. Treatment with a mean of $3.9 \pm 1.7$ antiarrhythmic drugs had been ineffective. Four of the patients in this study also were included in a prior study that described the results of focal ablation of atrial fibrillation. ${ }^{3}$

\section{Electrophysiologic Procedures}

The electrophysiologic procedures were performed with patients in the fasting state after informed consent was obtained. Except for one patient who had been treated with amiodarone, all antiarrhythmic drug therapy was discontinued at least five half-lives before the procedure. Multipolar electrode catheters were inserted into a femoral or subclavian vein and initially positioned in the high lateral right atrium, coronary sinus, and Hisbundle position. The catheter positioned near the His bundle later was used for mapping and ablation. This was a 6- or 7-French quadripolar electrode catheter that had a 4-mm distal electrode, an interelectrode spacing of 2-5-2 $\mathrm{mm}$, a deflectable tip, and a thermistor embedded in the distal electrode. Several ECG leads and the bipolar intracardiac electrograms were recorded on paper or optical disk. The filter settings for the intracardiac electrograms were 30 to $500 \mathrm{~Hz}$. Pacing was performed at twice diastolic threshold with a programmable stimulator using stimuli that had a duration of $2 \mathrm{msec}$.

\section{Mapping and Ablation}

In the four patients with persistent atrial fibrillation, internal cardioversion was performed on multiple occasions to allow mapping of the atrial premature depolarizations that initiated recurrent episodes of atrial fibril- lation. ${ }^{3}$ In four patients, frequent atrial premature depolarizations and nonsustained runs of atrial tachycardia or paroxysmal atrial fibrillation occurred spontaneously during the procedure. In the remaining patients, atrial premature depolarizations and runs of atrial tachycardia or atrial fibrillation were induced by infusion of isoproterenol or by rapid pacing to induce an initial episode of atrial fibrillation. Internal or transthoracic cardioversion was performed during the procedure if an episode of spontaneous or induced atrial fibrillation persisted for $>10$ minutes. The technique used for internal cardioversion has been described previously. ${ }^{5}$

Preliminary mapping in all patients indicated that the site of origin of the atrial premature depolarizations that were triggering episodes of atrial tachycardia or atrial fibrillation was not in the right atrium or superior vena cava. Mapping of the left atrium and pulmonary veins was performed in four patients through a patent foramen ovale and by transseptal catheterization in the remaining patients. A heparin bolus of 3,000 to 5000 units was administrated on entry into the left atrium, and an additional 1,000-unit bolus was administered every hour. Positioning of the ablation catheter within a pulmonary vein was verified in all patients by the fluoroscopic appearance of the ablation catheter outside of the cardiac silhouette, and by pulmonary vein angiography in 14 patients $(67 \%)$.

Radiofrequency energy was applied at target sites that were believed to be possible sites of origin based on relatively early local endocardial activation $(\geq 30 \mathrm{msec}$ earlier than the high right atrial electrogram). Radiofrequency energy was delivered at a cycle length of 500 to $550 \mathrm{kHz}$ by a generator that automatically adjusted the output to maintain a preset temperature of $52^{\circ}$ to $55^{\circ} \mathrm{C}$ at the electrode-tissue interface (EP Technologies, Sunnyvale, CA, USA; or Medtronic, Minneapolis, MN, USA). The applications were 30 to 60 seconds in duration.

A mean of $1.5 \pm 2$ (range 1 to 4 ) applications of radiofrequency energy were delivered within a pulmonary vein. Among the 23 effective ablation sites, 11 were located in the left superior pulmonary vein, 10 in the right superior pulmonary vein, and 2 in the left inferior pulmonary vein. Thirteen of the 23 effective ablation sites $(56 \%)$ were located in the main trunk of the pulmonary vein, and the remainder were located within a branch of the pulmonary vein, approximately 2 to $4 \mathrm{~cm}$ from the ostium. The mean duration of fluoroscopy was $68 \pm 42$ minutes (range 22 to 109 ). The only complication was a small pericardial effusion that resolved with conservative treatment in two patients.

\section{Analysis of Electrograms}

A total of 75 pulmonary venous sites were mapped, and these sites were classified into three categories for the purpose of electrogram analysis: (1) successful target sites (23 sites); (2) ineffective target sites within the pulmonary vein in which the successful target site was located (28 sites); and (3) sites within nonarrhythmogenic pulmonary veins, i.e., pulmonary veins other than 
the one in which the successful target site was located (24 sites).

Because the onset of the P wave on the surface ECG often was indistinct, endocardial activation times were measured relative to the high right atrial electrogram. A split electrogram was defined as an electrogram that had two discrete components separated by an isoelectric segment (Fig. 1).

\section{Statistical Analysis}

Continuous variables are expressed as mean $\pm 1 \mathrm{SD}$ and were compared using the Student's $t$-test or by analysis of variance. Discrete variables were compared using the Fisher exact test. $\mathrm{P}<0.05$ was considered statistically significant.

\section{Results}

\section{Split Potentials During Sinus Rhythm}

During sinus rhythm, a split electrogram was recorded at 29 of the 70 mapping sites $(39 \%)$. The two components of the split electrogram were separated by a mean of $22 \pm 27 \mathrm{msec}$. During sinus rhythm, there were no significant differences in the prevalence of split electrograms between the effective target sites, the ineffective target sites, and the sites in nonarrhythmogenic pulmonary veins (Table 1 ).

\section{Split Potentials During Atrial Premature Depolarizations}

The prevalence of split potentials during atrial premature depolarizations was significantly higher than during sinus complexes $(85 \%$ vs $39 \%$; $\mathrm{P}<0.05)$.

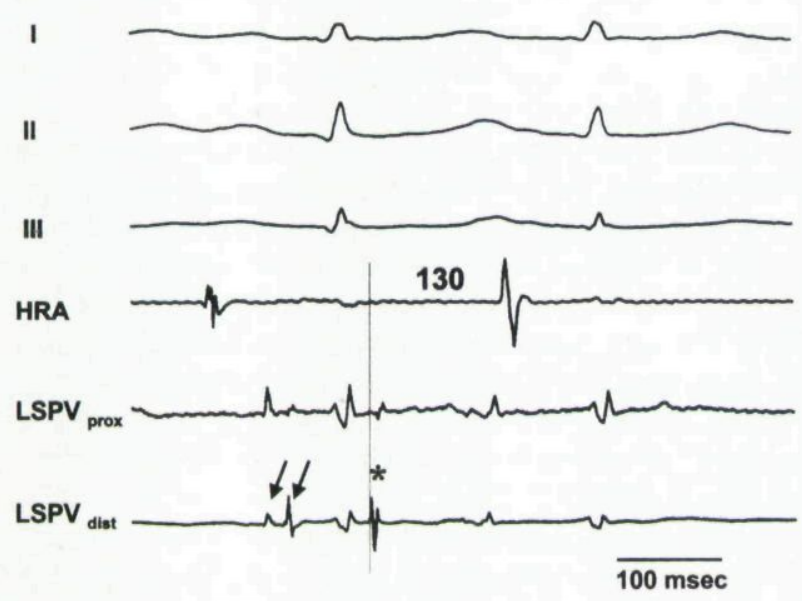

Figure 1. Example of a split electrogram during sinus rhythm and during atrial premature depolarization at a successful target site for ablation in the left superior pulmonary vein (LSPV). The arrows designate the two components of the split electrogram in the sinus beat. The endocardial activation time (relative to the high right atrial electrogram) of a premature depolarization (asterisk) arising in the LSPV is -130 msec. $H R A=$ high right atrial electrogram; $L S P V_{\text {prox }}$ and $L S P V_{\text {dist }}=$ recordings by the proximal and distal of electrodes of the ablation catheter in the LSPV.

\section{TABLE 1}

Prevalence of Split Potentials at Effective and Ineffective Ablation Sites Within Arrhythmogenic Pulmonary Veins and at Sites Within Nonarrhythmogenic Pulmonary Veins

\begin{tabular}{lccc}
\hline & $\begin{array}{c}\text { Effective } \\
\text { Sites } \\
(\mathbf{n}=\mathbf{2 3})\end{array}$ & $\begin{array}{c}\text { Ineffective } \\
\text { Sites } \\
(\mathbf{n}=\mathbf{2 8})\end{array}$ & $\begin{array}{c}\text { Other PVs } \\
(\mathbf{n}=\mathbf{2 4})\end{array}$ \\
\hline $\begin{array}{l}\text { Split potential } \\
\text { Sinus rhythm }\end{array}$ & $11(49 \%)$ & $11(39 \%)$ & $7(29 \%)$ \\
APD & $23(100 \%)$ & $25(89 \%)$ & $16(67 \%)^{*}$ \\
EAT relative to HRA & & & \\
$\quad$ (msec) & $129 \pm 66$ & $106 \pm 37$ & $100 \pm 20$ \\
$\begin{array}{l}\text { Sinus rhythm } \\
\text { APD }\end{array}$ & $-96 \pm 64$ & $-53 \pm 37^{*}$ & $-24 \pm 36^{*} \dagger$ \\
Onset of tachycardia & $-106 \pm 39$ & $-61 \pm 18^{*}$ & $-22 \pm 31^{*} \dagger$ \\
\hline
\end{tabular}

Continuous variables are expressed as mean $\pm 1 \mathrm{SD}$.

$* \mathrm{P}<0.05$ compared with effective sites; $\uparrow \mathrm{P}<0.05$ compared with ineffective sites.

$\mathrm{APD}=$ atrial premature depolarization; EAT = endocardial activation time; $\mathrm{HRA}=$ high right atrium; $\mathrm{PV}=$ pulmonary vein.

Split potentials were observed during atrial premature depolarizations at 23 effective target sites (100\%), at 25 ineffective target sites within the arrhythmogenic pulmonary vein $(89 \%)$, and at 16 sites within the nonarrhythmogenic pulmonary veins $(67 \%$; $\mathrm{P}<0.01)$.

During atrial premature depolarizations, the mean endocardial activation time at effective ablation site was $-96 \pm 64 \mathrm{msec}$, which was significantly earlier than the mean endocardial activation time at ineffective target sites within the arrhythmogenic pulmonary vein and earlier than at sites within nonarrhythmogenic pulmonary veins (Table 1).

\section{Electrograms at the Onset of Tachycardia or Atrial Fibrillation}

The onset of an episode of atrial tachycardia or atrial fibrillation was recorded at each of the 23 successful target sites. A tachycardia was recorded at 13 successful target sites, and 10 of these tachycardias degenerated into atrial fibrillation. Atrial fibrillation that was triggered by a premature depolarization within the pulmonary vein was recorded at ten successful target sites.

The mean endocardial activation time during atrial premature depolarizations that triggered atrial tachycardia or atrial fibrillation at successful target sites was significantly earlier than at ineffective target sites within the arrhythmogenic pulmonary vein and at sites within nonarrhythmogenic pulmonary veins (Fig. 2). An endocardial activation time of $-75 \mathrm{msec}$ or earlier relative to the high right atrial electrogram had a sensitivity of $83 \%$ and a specificity of $79 \%$ for the identification of a successful target site. Endocardial activation times earlier than -100 msec were recorded only at successful ablation sites, and endocardial activation times later than $-30 \mathrm{msec}$ were recorded only at sites within nonarrhythmogenic pulmonary veins (Fig. 2).

Within individual pulmonary veins, the endocardial activation time at the successful ablation site was always 




Figure 2. Comparison of endocardial activation times of premature pulmonary vein depolarizations recorded at 23 successful target sites, 28 unsuccessful target sites within the arrhythmogenic pulmonary vein, and 24 sites in nonarrhythmogenic pulmonary veins. Mean $\pm 1 S D$ is shown for each group. Mean endocardial activation times of the three groups all differed significantly from each other $(P<0.01)$.

earlier than at ineffective ablations sites. The mean difference between the endocardial activation times at successful and ineffective target sites within the same pulmonary vein was $50 \pm 25 \mathrm{msec}$ (range 20 to 110 ).

\section{Discussion}

\section{Main Finding}

The results of this study demonstrate that the endocardial activation time is helpful in identifying sites within pulmonary veins that initiate or generate atrial tachycardia/fibrillation. An endocardial activation time earlier than $-75 \mathrm{msec}$ relative to the high right atrial electrogram is approximately $80 \%$ sensitive and specific for an effective ablation site. Furthermore, an endocardial activation time earlier than $-100 \mathrm{msec}$ is specific to successful ablation sites, whereas endocardial activation times later than $-50 \mathrm{msec}$ are specific to unsuccessful ablation sites in the arrhythmogenic pulmonary vein and to sites within nonarrhythmogenic pulmonary veins.

\section{Split Potentials}

Previous experimental studies demonstrated that electrical depolarizations are able to propagate within pulmonary veins. ${ }^{6-9}$ Anatomically, the junction of the pul- monary veins and the left atrium is formed by extension of myocardial fibers from the atrial wall. These fibers are continuous in the adventitia and course in an oblique or spiral direction, forming a sphincter-like structure at the ostium of the pulmonary veins. ${ }^{10}$ The complex anatomic arrangement of these fibers within the pulmonary veins may lead to discontinuous propagation of a wave of depolarization and various conduction disturbances. ${ }^{11}$ This may explain the frequent occurrence of split electrograms within the pulmonary veins during sinus rhythm.

The results of this study demonstrate that split electrograms are ubiquitous within arrhythmogenic pulmonary veins. However, because they also are commonly recorded in nonarrhythmogenic pulmonary veins, split potentials are not of value in identifying successful ablation sites.

\section{Prior Studies}

Recent studies demonstrated that atrial tachycardia or atrial fibrillation may be initiated by premature depolarizations arising in a pulmonary vein or may be generated within a pulmonary vein. ${ }^{1-4}$ The results of the present study expand on the findings of these prior studies by providing a quantitative comparison of endocardial activation times at successful and unsuccessful target sites and in nonarrhythmogenic pulmonary veins, and suggest that the endocardial activation time is helpful in identifying an arrhythmogenic pulmonary vein.

\section{Limitations}

A limitation of this study is that not all pulmonary veins were systematically mapped in every patient. The reason for this was to minimize the duration of the procedures and the risk of complications.

In this study, because the onset of the P wave often was indistinct, the high right atrial electrogram was used as a reference for the endocardial activation time. Endocardial activation times determined relative to the $\mathrm{P}$ wave or some other point of reference would be different than when the high right atrial electrogram is used as the point of reference. Therefore, the findings of this study are valid only when the high right atrial electrogram is used as point of reference for determination of endocardial activation times.

None of the patients in this study developed clinical evidence of pulmonary vein stenosis during follow-up. However, because angiography was not performed during follow-up, the occurrence of asymptomatic pulmonary vein stenosis cannot be ruled out. Ablation techniques aimed at pulmonary vein isolation may be safer than focal ablation within a pulmonary vein, but this remains to be determined. Regardless of which ablation technique is found to be the safest, the results of this study should be helpful in identifying the site of origin of focal atrial fibrillation. 


\section{Conclusion}

During electrophysiologic procedures aimed at catheter ablation of atrial arrhythmias arising in a pulmonary vein, the endocardial activation time of the premature depolarizations that initiate the arrhythmias is useful in identifying successful ablation sites. The likelihood that a site of origin has been identified is greatest when the endocardial activation time precedes the high right atrial electrogram by $>100 \mathrm{msec}$. Conversely, when the endocardial activation time is $<30 \mathrm{msec}$ earlier than the high right atrial electrogram, the likelihood of successful ablation at that site is very low.

The results of this study demonstrate that in patients who have atrial arrhythmias triggered or generated within a pulmonary vein, split potentials are a nonspecific finding and are present not only at the successful target site, but also at other sites within the arrhythmogenic pulmonary vein and within nonarrhythmogenic pulmonary veins.

In this study, radiofrequency energy was delivered within the pulmonary vein, at the presumed site of origin of pulmonary vein depolarizations. To minimize the risk of pulmonary vein stenosis, the number of radiofrequency applications delivered within each vein was kept to a minimum, and there was no clinical evidence of pulmonary vein stenosis during follow-up. Another approach to ablation is isolation of an arrhythmogenic pulmonary vein with circumferential radiofrequency lesions at the ostium of the pulmonary vein..$^{12}$ The longterm efficacy and safety of focal ablation within a pulmonary vein compared with pulmonary vein isolation remain to be determined.

\section{References}

1. Jaïs P, Haïssaguerre M, Shah DC, et al: A focal source of atrial fibrillation treated by discrete radiofrequency ablation. Circulation 1997;95:572-576.

2. Haïssaguerre M, Jaïs P, Shah DC, et al: Spontaneous initiation of atrial fibrillation by ectopic beats originating in the pulmonary veins. N Engl J Med 1998;339:659-666.

3. Lau CP, Tse HF, Ayers GM: Defibrillation-guided radiofrequency ablation of atrial fibrillation secondary to an atrial focus. J Am Coll Cardiol 1999;33:1217-1226.

4. Hsieh MH, Chen SA, Tai CT, et al: Double multielectrode mapping catheters facilitate radiofrequency catheter ablation of focal atrial fibrillation originating from pulmonary veins. J Cardiovasc Electrophysiol 1999;10:136-144.

5. Lok NS, Lau CP, Ho DSW, et al: Hemodynamic effects and clinical determinants of defibrillation threshold for low energy transvenous atrial defibrillation in patients with chronic atrial fibrillation. PACE 1997:20:899-908.

6. De Almeida OA, Bohm GM, De Paula Carvalho M, et al: The cardiac muscle in the pulmonary vein of rat: A morphological and electrophysiological study. J Morph 1975;135:409-434.

7. Cheung DW: Electrical activity of the pulmonary vein and its interaction with the right atrium in the guinea-pig. J Physiol 1981;314:445-456.

8. Zipes DP, Knope RF: Electrical properties of the thoracic vein. Am J Cardiol 1972;29:372-376.

9. Spach MS, Barr RC, Jewett PH: Spread of excitation from the atrium into thoracic veins in human beings and dogs. Am J Cardiol 1972;30:844-854.

10. Nathan $H$, Eliakim M: The junction between the left atrium and the pulmonary veins: An anatomic study of human hearts. Circulation $1966 ; 34: 412-422$.

11. Ito M, Arita M, Saeki K, et al: Functional properties of sinocaval conduction. Jpn J Physiol 1967;17:174-189.

12. Schwartzman D: Circumferential radiofrequency ablation of pulmonary vein orifices: Feasibility of a new technique. (Abstract) PACE 1999;22:711. 
Copyright of Journal of Cardiovascular Electrophysiology is the property of Blackwell Publishing Limited and its content may not be copied or emailed to multiple sites or posted to a listserv without the copyright holder's express written permission. However, users may print, download, or email articles for individual use. 